M. OZAWA

KODAI MATH. J.

3 (1980), 310-314

\title{
ON THE BOUNDEDNESS OF THE SOLUTIONS OF A DIFFERENTIAL EQUATION IN THE COMPLEX DOMAIN
}

\author{
By Mitsuru Ozawa
}

1. In our previous paper [1] we proved a boundedness criterion for every solution of $w^{\prime \prime}+F(z) w=0$ along a ray. In this paper we shall give an extension of our earlier result. The result which we want to prove is the following

THEOREM 1. Let $F(z)$ be $g(r) e^{i \gamma(r)}$ along the ray $l: r e^{i 0}(\theta:$ fixed $)$ such that $X(r)=g(r) \cos (\gamma(r)+2 \theta)$ is monotone increasing for $r \geqq r_{0}, X\left(r_{0}\right)>0$ and there is $a$ positive constant $K$ such that

for $t \geqq r_{0}$ and

$$
|Y(t)| \leqq K X^{\prime}(t)
$$

$$
\int^{\infty}|Y(t)| X(t)^{K} d t<\infty
$$

where $Y(r)=g(r) \sin (\gamma(r)+2 \theta)$. Further assume that $F(z)$ is regular around the ray $l$. Then every solution of $w^{\prime \prime}+F(z) w=0$ is bounded along the ray $l$.

As an application of the above theorem we shall prove the following

THEOREM 2. Under the same notations as in the above theorem assume that there is a positive constant $K$ such that

for $t \geqq r_{0}, X\left(r_{0}\right)>0$,

$$
|Y(t)| \leqq K X^{\prime}(t)
$$

$$
\int^{\infty}|Y(t)| d t<\infty
$$

and $g(r)$ is bounded along the ray $l$. Further assume that $F(z)$ is regular around the ray $l$. Then every solution of $w^{\prime \prime}+F(z) w=0$ is bounded along $l$ and the same is true for its derivative.

2. Proof of Theorem 1. For completeness we shall give its full proof here. Let us put $w(z)=R(r) \exp (i \Theta(r))$ along $l$. Then the differential equation

Received May 9, 1979 
$w^{\prime \prime}+F(z) w=0$ gives

(1)

$$
\left\{\begin{array}{l}
R^{\prime \prime}(r)+\left\{X(r)-\Theta^{\prime}(r)^{2}\right\} R(r)=0, \\
\left\{\Theta^{\prime}(r) R(r)^{2}\right\}^{\prime}+Y(r) R(r)^{2}=0 .
\end{array}\right.
$$

Let us consider the following quadratic functional

Then

$$
2 H=R^{\prime 2}+R^{2} \Theta^{\prime 2}+X R^{2} .
$$

$$
\begin{aligned}
2 H^{\prime} & =2 R^{\prime} R^{\prime \prime}+2 R^{2} \Theta^{\prime} \Theta^{\prime \prime}+2 R R^{\prime} \Theta^{\prime 2}+X^{\prime} R^{2}+2 X R R^{\prime} \\
& =X^{\prime} R^{2}-2 Y \Theta^{\prime} R^{2}
\end{aligned}
$$

by the equation (1). By integration from $r_{1}$ to $r$ we have

$$
2 H(r)=2 H\left(r_{1}\right)+\int_{r_{1}}^{r} X^{\prime} R^{2} d t-2 \int_{r_{1}}^{r} Y \Theta^{\prime} R^{2} d t
$$

that is,

$$
\begin{aligned}
& R^{\prime}(r)^{2}+R(r)^{2} \Theta^{\prime}(r)^{2}+X(r) R(r)^{2} \\
& =R^{\prime}\left(r_{1}\right)^{2}+R\left(r_{1}\right)^{2} \Theta^{\prime}\left(r_{1}\right)^{2}+X\left(r_{1}\right) R\left(r_{1}\right)^{2} \\
& \quad+\int_{r_{1}}^{r} R^{2} d X-2 \int_{r_{1}}^{r} Y \Theta^{\prime} R^{2} d t .
\end{aligned}
$$

Now we shall estimate the last integral. By the second equation of (1)

$$
-\int_{r_{1}}^{r} Y R^{2} d t=\Theta^{\prime}(r) R(r)^{2}-\Theta^{\prime}\left(r_{1}\right) R\left(r_{1}\right)^{2}
$$

Hence

$$
\begin{aligned}
& -\int_{r_{1}}^{r} Y(t) \Theta^{\prime}(t) R(t)^{2} d t \\
& =-\Theta^{\prime}\left(r_{1}\right) R\left(r_{1}\right)^{2} \int_{r_{1}}^{r} Y(t) d t+\int_{r_{1}}^{r} Y(l) \int_{r_{1}}^{t} Y(s) R(s)^{2} d s d t .
\end{aligned}
$$

Therefore

$$
\left|-\int_{r_{1}}^{r} Y \Theta^{\prime} R^{2} d t\right| \leqq\left|\Theta^{\prime}\left(r_{1}\right)\right| R\left(r_{1}\right)^{2} \int_{r_{1}}^{r}|Y(t)| d t+\int_{r_{1}}^{r}|Y(t)| R(t)^{2} d t \int_{r_{1}}^{r}|Y(t)| d t .
$$

By the assumption

$$
\int^{\infty}|Y| X^{K} d t<\infty
$$

and by $X(r) \geqq X\left(r_{1}\right)>0$ for $r \geqq r_{1}>r_{0}$,

$$
\int_{r_{1}}^{\infty}|Y| d t \leqq \frac{1}{X\left(r_{1}\right)^{K}} \int_{r_{1}}^{\infty}|Y| X^{K} d t<\infty
$$

We set 


$$
C_{0}=\int_{r_{1}}^{\infty}|Y(t)| d t<\infty
$$

Hence

$$
\left|-\int_{r_{1}}^{r} Y \Theta^{\prime} R^{2} d t\right| \leqq C_{0}\left|\Theta^{\prime}\left(r_{1}\right)\right| R\left(r_{1}\right)^{2}+C_{0} \int_{r_{1}}^{r}|Y| R^{2} d t .
$$

By $|Y(t)| \leqq K X^{\prime}(t)$ for $t \geqq r_{0}$

$$
C_{0} \int_{r_{1}}^{r}|Y(t)| R(t)^{2} d t \leqq C_{0} K \int_{r_{1}}^{r} R(t)^{2} d X(t)
$$

Thus by (2)

$$
\frac{1}{2} X(r) R(r)^{2} \leqq C_{1}+\frac{1}{2}\left(1+2 C_{0} K\right) \int_{r_{1}}^{r} R(t)^{2} d X(t)
$$

with a positive constant $C_{1}$. By the same process as in the proof of the Gronwall inequality we have

that is,

$$
X(r) R(r)^{2} \leqq 2 C_{1} X(r)^{1+2 C_{0} K} X\left(r_{1}\right)^{-1-2 C_{0} K},
$$

$$
R(r)^{2} \leqq C^{*} X(r)^{2 C_{0} K^{*}}
$$

with a positive constant $C^{*}$, which depends on $r_{1}$. If $X(r)$ is bounded, then $R(r)$ is bounded by (3). Hence we may assume that $X(r)$ is unbounded. Since $X(r)$ is non-decreasing, we may assume that $X(r)$ is larger than 1 for $r \geqq r_{0}$. We now take an $r_{1}$ sufficiently large so that $2 C_{0} \leqq 1$, which is clearly possible. Then

$$
\begin{aligned}
\left|\Theta^{\prime}(r) R(r)^{2}-\Theta^{\prime}\left(r_{1}\right) R\left(r_{1}\right)^{2}\right| & \leqq \int_{r_{1}}^{r}|Y| R^{2} d t \\
& \leqq C^{*} \int_{r_{1}}^{r}|Y(t)| X(t)^{2 C_{0} K} d t \\
& \leqq C^{*} \int_{r_{1}}^{r}|Y(t)| X(t)^{K} d t \leqq C_{2} .
\end{aligned}
$$

Hence

$$
\left|\Theta^{\prime}(r)\right| R(r)^{2} \leqq C_{2}+\left|\Theta^{\prime}\left(r_{1}\right)\right| R\left(r_{1}\right)^{2}=C_{3}
$$

This implies that

$$
\left|\int_{r_{1}}^{r} Y \Theta^{\prime} R^{2} d t\right| \leqq C_{3} \int_{r_{1}}^{\infty}|Y| d t=C_{0} C_{3}
$$

Therefore

$$
X(r) R(r)^{2} \leqq D+\int_{r_{1}}^{r} R(t)^{2} d X(t)
$$

with a suitable constant $D>0$. By the Gronwall inequality $R(r)^{2} \leqq D / X\left(r_{1}\right)$, which is just the desired result. 
3. Proof of Theorem 2. The inequality (3) holds in this case too. Since $X(r)$ is monotone increasing and $X\left(r_{0}\right)>0$ and since $g(t)$ is bounded and $X(t) \leqq$ $g(t)$ for $t \geqq r_{0}, X(t)$ is bounded. Hence (3) implies the boundedness of $R$, which is the first desired result. For the second half

$$
\frac{1}{2}\left(1+2 C_{0} K\right) \int_{r_{1}}^{r} R(t)^{2} d X(t) \leqq C^{*}\left\{X(r)^{1+2 C_{0} K}-X\left(r_{1}\right)^{1+2 C_{0} K}\right\} .
$$

by (3). The right hand side term is bounded along the ray $l$. Therefore

is bounded. Hence

$$
R^{\prime}(r)^{2}+R(r)^{2} \Theta^{\prime}(r)^{2}+X(r) R(r)^{2}
$$

$$
\left|w^{\prime}(z)\right|=\left|R^{\prime}(r)+\imath R(r) \Theta^{\prime}(r)\right|
$$

is bounded along the ray $l$.

A remark should be mentioned here. $X(r) \rightarrow b$ as $r \rightarrow \infty$. By

$$
\int^{\infty}|Y(t)| d t<\infty
$$

$|b||\sin (\gamma(r)+2 \theta)| /|\cos (\gamma(r)+2 \theta)| \rightarrow 0$ as $r \rightarrow \infty$. Since $b>0, \quad \sin (\gamma(r)+2 \theta) \rightarrow 0$ and $\cos (\gamma(r)+2 \theta) \rightarrow 1$ as $r \rightarrow \infty$. Hence $g(r) \rightarrow b$, that is, $|F(z)| \rightarrow b$ along the ray $l$. By the way in the case of Theorem 1 we can say that $\left|w^{\prime}(z)\right|^{2} / X(r)$ is bounded along the ray $l$.

4. Taam's result. In this section we shall give a shorter proof of Taam's result [2]. There is no new idea. Let us consider the following functional

$$
H=b R^{2}+R^{\prime 2}+\Theta^{\prime 2} R^{2},
$$

where $b$ is a positive constant. Then

$$
\frac{d}{d r} H=2 b R R^{\prime}+2 R^{\prime} R^{\prime \prime}+2 \Theta^{\prime} \Theta^{\prime \prime} R^{2}+2 \Theta^{\prime 2} R R^{\prime} .
$$

By (1) we have

Hence

$$
H^{\prime}=2(b-X) R R^{\prime}-2 Y \Theta^{\prime} R^{2} .
$$

$$
\begin{aligned}
H^{\prime} & \leqq\left\{|b-X|\left(b R^{2}+R^{\prime 2}\right)+|Y|\left(\Theta^{\prime 2} R^{2}+b R^{2}\right)\right\} \frac{1}{\sqrt{b}} \\
& \leqq \frac{1}{\sqrt{b}}(|b-X|+|Y|) H .
\end{aligned}
$$

Therefore

If

$$
H(r) \leqq H\left(r_{1}\right) \exp \frac{1}{\sqrt{ } b} \int_{r_{1}}^{r}\{|b-X|+|Y|\} d t
$$

$$
\int^{\infty}(|b-X|+|Y|) d t<\infty
$$


then $w$ and $w^{\prime}$ are bounded along the ray $l$. This is nothing but a result due to Taam.

5. Next we start from the following quadratic functional

$$
Q=\sqrt{ } X R^{2}+\frac{1}{\sqrt{X}}\left(R^{\prime 2}+R^{2} \Theta^{\prime 2}\right) .
$$

By the equation (1)

$$
Q^{\prime}=\frac{1}{2} \frac{X^{\prime}}{\sqrt{X}} R^{2}-\frac{1}{2} \underset{\sqrt{ } X^{3}}{X^{\prime}}\left(R^{\prime 2}+R^{2} \Theta^{\prime 2}\right)-2 \underset{\sqrt{ } X}{Y} \Theta^{\prime} R^{2} .
$$

Now the last term is estimated by

$$
\frac{|Y|}{\sqrt{X^{3}}}\left(\frac{1}{a} X^{\alpha} \Theta^{\prime 2} R^{2}+a X^{\beta} R^{2}\right)
$$

with a positive constant $a$ and constants $\alpha, \beta$ satisfying $\alpha+\beta=2,0 \leqq \alpha \leqq 2$. Assume that $X^{\prime} \geqq 2|Y| X^{\alpha} / a$ and $X(t)>0$ for $t \geqq r_{0}$. Then

$$
Q^{\prime} \leqq \frac{1}{2} \frac{X^{\prime}}{\sqrt{X}} R^{2}\left(1+a^{2} X^{3-1-a}\right)
$$

and hence with a positive constant $C$

$$
\sqrt{ } X(r) R(r)^{2} \leqq C+\frac{1}{2} \int_{r_{1}}^{r} X^{\prime} X^{2}\left(1+a^{2} X^{, j-1-n}\right) d t .
$$

Thus

$$
R(r)^{2} \leqq C \frac{1}{\sqrt{X\left(r_{1}\right)}} \exp \frac{a^{2}}{2} \int_{r_{1}}^{r} X^{\beta-2-\alpha} X^{\prime} d t
$$

Assume that $\alpha>1 / 2$. Then $-\gamma=\beta-1-\alpha<0$. In this case

$$
\begin{aligned}
R(r)^{2} & \leqq \frac{C}{\sqrt{ } X\left(r_{1}\right)} \exp \left\{\frac{a^{2}}{2 \gamma}\left(X\left(r_{1}\right)^{-\ddot{i}}-X(r)^{-\check{r}}\right)\right\} \\
& \leqq B .
\end{aligned}
$$

Thus we have the following

Theorem 3. Suppose that $X^{\prime} \geqq 2|Y| X^{\alpha} / a$ with positive constants $a$ and $\alpha$, $2 \geqq \alpha>1 / 2$ and $x(t)>0$ for $t \geqq r_{0}$. Then every solution $w$ of $w^{\prime \prime}+F(z) u=0$ is bounded along the ray $l$.

\section{REFERENCES}

11 OzAwA, M. On a solution of $w^{\prime \prime}+e^{-z} w^{\prime}+(a z+b) w=0$. Kodai Math. J. 3 (1980), 295-309.

[2] ТААм, C.-T. The boundedness of the solutions of a differential equation in the complex domain. Pacific J. Math. 2 (1952), 643 654.

Department of Mathematics,

Tokyo Institute of TechNology. 